OPEN ACCESS

Edited by:

Sanket J. Joshi,

Sultan Qaboos University, Oman

Reviewed by:

Alexei E. Solovchenko,

Lomonosov Moscow State University,

Russia

Jaoon Young Hwan Kim,

National Marine Biodiversity Institute

of Korea, South Korea

*Correspondence:

Fanping Meng

mengfanping@ouc.edu.cn

Specialty section:

This article was submitted to

Microbiotechnology,

a section of the journa

Frontiers in Microbiology

Received: 06 July 2021

Accepted: 30 July 2021

Published: 18 August 2021

Citation:

Zhang B, Wu J and Meng F (2021) Adaptive Laboratory Evolution

of Microalgae: A Review of the

Regulation of Growth, Stress Resistance, Metabolic Processes, and Biodegradation of Pollutants.

Front. Microbiol. 12:737248. doi: 10.3389/fmicb.2021.737248

\section{Adaptive Laboratory Evolution of Microalgae: A Review of the Regulation of Growth, Stress Resistance, Metabolic Processes, and Biodegradation of Pollutants}

\author{
Bo Zhang ${ }^{1,2}$, Jiangyue $\mathrm{Wu}^{3}$ and Fanping Meng ${ }^{1,2 *}$ \\ 'Key Laboratory of Marine Environment and Ecology, Ministry of Education, Ocean University of China, Qingdao, China, \\ ${ }^{2}$ College of Environmental Science and Engineering, Ocean University of China, Qingdao, China, ${ }^{3}$ National Marine Hazard \\ Mitigation Service, Ministry of Natural Resource of the People's Republic of China, Beijing, China
}

Adaptive laboratory evolution (ALE) experiments are a serviceable method for the industrial utilization of the microalgae, which can improve the phenotype, performance, and stability of microalgae to obtain strains containing beneficial mutations. In this article, we reviewed the research into the microalgae ALE test and assessed the improvement of microalgae growth, tolerance, metabolism, and substrate utilization by ALE. In addition, the principles of ALE and the key factors of experimental design, as well as the issues and drawbacks of the microalgae ALE method were discussed. In general, improving the efficiency of ALE and verifying the stability of ALE resulting strains are the primary problems that need to be solved in future research, making it a promising method for the application of microalgae biotechnology.

Keywords: adaptive laboratory evolution, microalgae, growth, metabolic regulation, biodegradation of pollutants, stress resistance

\section{INTRODUCTION}

Microalgae are of importance in biotechnological applications, ranging from biofuel production, production of high-value by-products, bioremediation of pollutants, by virtue of their rapid growth rate, high photosynthetic efficiency, acceptable adaptability, and eco-friendly nature (Mohsenpour et al., 2021). Industrial microalgae can often withstand certain unfavorable environmental conditions such as high concentrations of phenol (Wang et al., 2016; Li et al., 2021) and flue gas (Li T. et al., 2016; Cheng et al., 2019). This highlights the significance of enhancing the environmental tolerance of microalgae before application. To date, it has been difficult to improve the specific phenotype of microalgae by genome editing technology, as the tolerance is not commonly controlled by a single gene, and most microalgae have no sequenced genome or accurate gene annotation (Zhao and Huang, 2020). On the contrary, Adaptive laboratory evolution (ALE) is a feasible method based on stress induction, which can improve the tolerance of microalgae under specific environmental stress (Zchut et al., 2003; Sandberg et al., 2019).

ALE is an attractive technique for improving the performance of microorganisms, optimizing strain phenotypes, ascertaining biological phenomena caused by the stress conditions, and latent pathway activation (Wang et al., 2016; Xiong et al., 2017; Li et al., 2021; Okurowska et al., 2021). 
ALE experiments began with a controlled evolution experiment on bacteria around 1950 (Novick and Szilard, 1950; Atwood et al., 1951). Thereafter, Bennett et al. (1992) and Bennett and Lenski (1993). selected Escherichia coli as the model organism, and successfully obtained a strain that can adapt to temperatures between 32 and $42^{\circ} \mathrm{C}$ after 2000 generations. In 1991, the temperature ALE tests of Lomentaria baileyana and Lomentaria orcadensis were conducted for more than 4 months. The results showed that the difference in lowtemperature tolerance between the two species was caused by the difference in photoinhibition sensitivity, and there were differences in the high-temperature stability of electron transport or energy transport between the two species (Kuebler et al., 1991). Furthermore, microorganisms can divide rapidly to achieve a high cell density, and the higher mutation rate and small genome sizes mean that their mutation rates are extremely high (Gresham and Dunham, 2014). Given the speed with which beneficial mutations can arise and become fixed, the simplest way of ALE is to extend the cell culture period in the selected environment to obtain beneficial mutations by natural selection. Many research processes have "adaptively evolved" their laboratory microorganisms merely through the inevitable growth or plate inoculation cycle in cell culture. Profiting from the development of proteomics (Wang et al., 2018), transcriptomics (Perrineau et al., 2014b; Li K. et al., 2017; Qi et al., 2017; Zhou et al., 2017; Li X. et al., 2018; Sun et al., 2018a; Cheng et al., 2019; Zhu et al., 2020; Zhang et al., 2021), genomics (Perrineau et al., 2014a; Helliwell et al., 2015; Shin et al., 2017; Rossoni and Weber, 2019; Yu et al., 2020), and metabolomics (Lohbeck et al., 2014; Li X. et al., 2017; Hu et al., 2020), the application of appropriate bioinformatics techniques will contribute to identification of the key mutation mechanisms in ALE (Sandberg et al., 2019).

In this review we summarize the applications of ALE in microalgae, analyze the improvement of ALE in microalgal growth, tolerance, metabolism, and substrate utilization, highlighting several typical studies, summarizing some adaptive mechanisms, and discussing the future direction of ALE in microalgae. The coupling strategy of ALE and microalgae may provide a costeffective, efficient approach for the practical industrial application of microalgae.

\section{ALE PRINCIPLE AND EXPERIMENTAL DESIGN}

In the preceding ALE studies, researchers often used the terms acclimation and adaptation indiscriminately. Ownby et al. (2002) defined these two terms in the study on the tolerance of fish (Fundulus heteroclitus) to creosote-contaminated sediments. Recent studies on ALE also try to provide a clear definition (Kleine et al., 2021; LaPanse et al., 2021). In conclusion, acclimation is a type of phenotypic plasticity, not heritable, that organisms adapt to the environment rapidly in a short time; adaptation involves the acquisition or recombination of genetic traits, which can enhance the phenotype of multiple generations to applied stress.

Mutations are the basis for acquiring beneficial mutants through ALE. According to previous studies, experimental measures of mutation rates in microalgae range from $10^{-5}$ to $10^{-10}$ mutants per cell per generation (Marvá et al., 2010; Huertas et al., 2011; Perrineau et al., 2014a). Various types of mutations, include transposable element (insertional sequence, IS), single-nucleotide polymorphisms (SNPs), and insertions and deletions (InDels). By integrating a type-II Gulliver-related transposable element into the $\mathrm{B}_{12}$-independent methionine synthase gene (METE) during $\mathrm{ALE}, C$. reinhardtii became $\mathrm{B}_{12}$ dependent in fewer than 500 generations of growth in the presence of vitamin $B_{12}$ (Helliwell et al., 2015). After 1880 generations of ALE, 1937 polymorphic DNA regions were found, among which 149 SNPs resulted in nucleotide substitution in the fast-growing C. reinhardtii genome (Perrineau et al., 2014a). Changes in these genomes can alter the metabolic pathways of mutant strains, making it easier for the resulting strains to adapt to the imposed environmental conditions. The difference between ALE and natural selection is that ALE can obtain target phenotypes by controlling specific environmental conditions. In the early stage of ALE, mutants induced by specific conditions began to appear in the cultures. Over time, these beneficial mutants gradually replaced the original algal strain, eventually becoming the dominant strain.

At present, the number of ALE studies on freshwater species is much greater than that on marine species (Supplementary Table 1). Several studies on the ALE of microalgae under synthetic wastewater (Rezaei et al., 2019), municipal secondary-treated wastewater (Osundeko et al., 2014), light intensity stress (Deblois et al., 2013), and nitrogen stress (Li T. et al., 2016) proved that the effect of ALE on microalgal fitness is species-specific. Unlike a chemostat, which automatically performs continuous adaptive evolution, as commonly used in bacteria and fungi, microalgae perform ALE frequently by way of a serial transfer procedure. Each transfer procedure is considered to have completed a cycle, and one generation indicates that the number of microalgal cells has doubled. In general, a single cycle of ALE of microalgae lasted from 1 to 30 days. The applied stress conditions limited the density of algal cells at the end of each cycle, resulting in a lower number of generations in each cycle. Undoubtedly, the number of generations is more important than cycle when reaching a more stable adaptive evolutionary algal strains, but this datum is usually lacking in most cases, and should be elucidated in future research (Supplementary Table 1). In fact, the selection of environmental stress determines the efficiency of ALE to a certain extent (Figure 1). If the environmental stress exceeds the tolerance of microalgae, microalgae cannot survive, it will lead to the failure of ALE (Bautista-Chamizo et al., 2018). If the intensity of environmental stress is very low, the efficiency of ALE will be low, and the whole process will be time-consuming (Cheng et al., 2019). Similarly, the design of cycle times of ALE also affects the evolution efficiency and robustness of microalgae. For instance, the ALE of C. reinhardtii in TAP medium increased the biomass concentration of the endpoint strains of cc4324, 
cc4326, and cc4334, which gradually appeared after 15 cycles (Yu et al., 2013). Furthermore, the results of several studies indicated that the biomass concentration of different species of microalgae increased gradually after 15 or 16 cycles during the evolution, indicating that the growth of microalgae was unstable during the early stage of ALE (Fu et al., 2012, 2013; Yu et al., 2013).

To optimize the fitness of microalgae under multiple stresses, some studies have examined two-step ALE: after 210 cycles of ALE with sethoxydim, sesamol-based ALE (over 100 cycles) was undertaken, to increase DHA productivity of C. cohnii (Diao et al., 2019). In addition, Chlorella sp. AE10 with high $\mathrm{CO}_{2}$ tolerance was obtained by implementing ALE (Li et al., 2015). Thereafter, the second ALE was implemented to improve the tolerance of Chlorella sp. AE10 in a high-salinity environment (30 g/L NaCl) (Li X. et al., 2018). Besides, a novel two-step ALE method combining ${ }^{60} \mathrm{Co}-\gamma$ irradiation (Cheng et al., 2016) and high concentrations of $\mathrm{CO}_{2}$ (Cheng et al., 2016; Kato et al., 2017) has also been proposed to improve the efficiency of ALE. Most studies only apply a single stress condition at any time to improve the tolerance of microalgae to the stress exerted, but practical application conditions are far less suitable for the growth of microalgae than laboratory conditions, and multiple stresses may exist at the same time. Accordingly, multiple stress conditions can be applied simultaneously in an ALE experiment to improve the efficiency of ALE to cope with the complex environmental conditions encountered in practice. Unfortunately, only three studies describe the application of cooperative two-factor ALE strategy: one to optimize the lipid productivity of Schizochytrium sp. in combination with low temperature and high salinity (Sun et al., 2018b), another to improve growth and tolerance of Emiliania huxleyi under $\mathrm{CO}_{2}$ and high-temperature stresses (Schlüter et al., 2014), and the other to promote growth of Thalassiosira weissflogii by combining high temperature and high $\mathrm{CO}_{2}$ concentration at the expense of inhibiting photochemical performances (Zhong et al., 2021).

Part of the current research is to determine an ALE time endpoint, and some terminate the experiment based on changes in the phenotype of the algal strain. Although ALE can be implemented indefinitely, for practical application, it is necessary to determine a reasonable ALE endpoint by comparing the potential benefits of algal fitness with the time and economic cost of ALE. In addition, modeling methods have been used to optimize the ALE on bacteria and determine the optimal endpoint (Bittihn et al., 2017; LaCroix et al., 2017), which will also be a dilemma that needs to be solved in a subsequent study.

\section{ALE APPLICATION IN ENHANCING MICROALGAL GROWTH}

The industrial application of microalgae commonly under the abiotic stress, such as nitrogen stress, high light intensity, and osmotic stress, may increase the content of high value-added metabolites in microalgae. Nonetheless, the stresses typically inhibit the growth of microalgae, by that reducing their overall productivity, which increases the cost of microalgae technology application. ALE is a stress-induced procedure to optimize the tolerance of microalgae to the applied stress. Accordingly, ALE has been applied to optimize the growth of varied industrially related microalgae, to optimize the growth of microalgae under specific conditions, and to reveal the latent mechanism of ALE in microalgal growth optimization (Yu et al., 2013; Wang et al., 2016; Li X. et al., 2017; Kim et al., 2021; Li et al., 2021). Since the growth rate of microalgae is a primary consideration for the successful application of microalgae related biotechnology, the characterization of microalgal growth is usually one of the indicators evaluated in an ALE. For example, Shin et al. (2017) in the process of maintaining the growth of Chlamydomonas reinhardtii CC-124 for 5 years, inadvertently conducted an ALE experiment, and isolated a mutant strain with increased growth rate and lipid production. This rapid-growth phenotype was achieved through non-synonymous substitution to 33 genes which will be involved in cell cycle, division, or proliferation. Another study indicated that $C$. reinhardtii CC-503 evolved after 1880 generations under continuous illumination, the growth rate of evolved strain was 1.35 times that of the original strain, which was caused by 149 single nucleotide polymorphisms issuing non-synonymous amino acid substitutions and up-regulation of genes involved in protein synthesis, the cell cycle, and cellular respiration (Perrineau et al., 2014a). The results of these two cases suggest that continuous illumination can induce the strains derived from C. reinhardtii to achieve rapid growth rates via nonsynonymous substitutions and up-regulation of gene expression related to cell proliferation.

ALE has been used to improve growth capabilities of Haematococcus pluvialis under $15 \% \quad \mathrm{CO}_{2}$ (Cheng et al., 2016). Transcriptome sequencing analysis indicated that ALE enhanced photosynthesis, carbon fixation, and glycolysis pathway of $H$. pluvialis. The up-regulation of $P e t H$ (ferredoxin$\mathrm{NADP}^{+}$reductase) in electron transportation, ATPFOA (F-type $\mathrm{H}^{+}$-transporting ATPase subunit a) in ATP synthase, and PetJ (cytochrome c6) in NADPH generation promoted photosynthesis. The up-regulation of genes of C3 and C4 pathways ( $P d d K$, pyruvate, orthophosphate dikinase; $F B A$, fructose-bisphosphate aldolase, class I) helped increase carbon fixation under a high concentration of $\mathrm{CO}_{2}$ ( $\mathrm{Li} \mathrm{K}$. et al., 2017).

\section{ALE APPLICATION IN IMPROVING STRESS RESISTANCE OF MICROALGAE}

ALE has been proved to improve the tolerance of microalgae to stress. ALE researchers have revealed the adaptive effects under various conditions: flue gas (mainly includes $\mathrm{CO}_{2}, \mathrm{NO}_{x}$, and $\mathrm{SO}_{x}$ ) (Cheng et al., 2019), ultraviolet radiation (UVR) (Korkaric et al., 2015), light intensity (Deblois et al., 2013), organic contaminants (Cho et al., 2016; Wang et al., 2016; Li et al., 2021), wastewater (the main stressors were high concentrations of heavy metals, oxygen, and ammonium nitrogen) (Osundeko et al., 2014), landfill leachate (the main stressors were high concentrations of heavy metals, and ammonium nitrogen) (Okurowska et al., 2021), heavy metals (Muyssen and Janssen, 2001; Mikulic and Beardall, 2014; Yu et al., 2020), nutrient stress (Helliwell et al., 2015; Li T. et al., 2016), sludge extract 


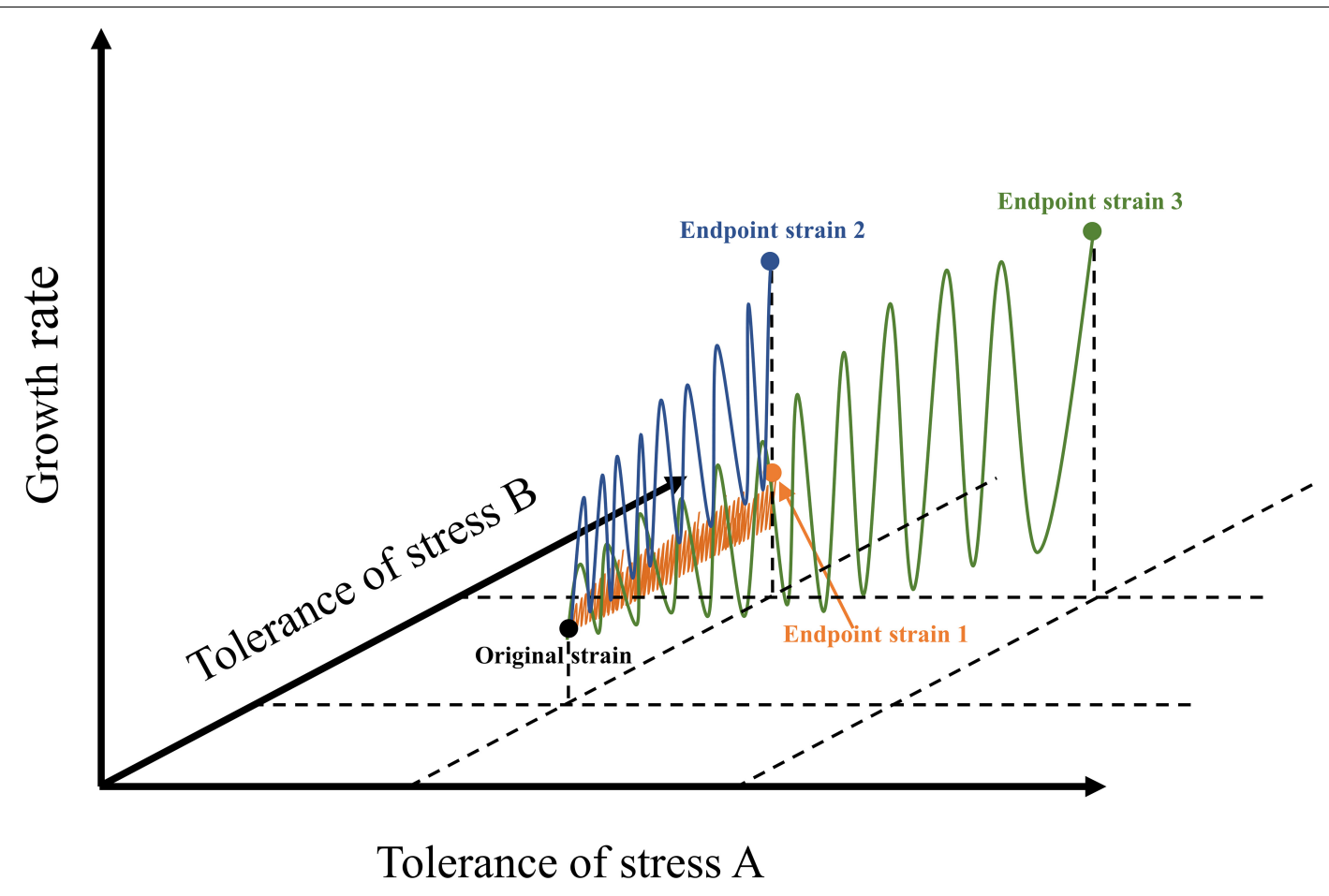

FIGURE 1 | Schematic diagram of different microalgal ALE approaches. The blue line represents ALE pattern of microalgae under appropriate stress concentration; the orange line indicates the ALE pattern of microalgae under low stress concentration; the green line represents the possible ways in which ALE of microalgae could be applied to two stress conditions simultaneously. This figure was modified according to the research (Zhao and Huang, 2020).

(Wang et al., 2018), $\mathrm{CO}_{2}$ (Collins and Bell, 2004; Lohbeck et al., 2012; Schlüter et al., 2014; Li et al., 2015; Cheng et al., 2016; Schaum et al., 2017; Listmann et al., 2020), and high salinity (Perrineau et al., 2014b; Lachapelle et al., 2015; Kato et al., 2017; Meijer et al., 2017; Li X. et al., 2018; Sun et al., 2018a; Hu et al., 2020). The cultivation of microalgae under abiotic stress conditions may increase the synthesis of high-value metabolites. However, these compounds generally inhibit cell growth, thereby reducing the overall productivity of microalgae and increasing the cost of production. As a result, the efficiency of practical application of most microalgae-based engineering techniques is often limited due to abiotic stress. For example, the toxicity of high concentrations of phenol prevents algae from being able to degrade phenol, which limits the application of microalgaebased bioremediation technology in the case of phenol leakage. Li et al. (2021) addressed this with an ALE experiment on Isochrysis galbana in $200 \mathrm{mg} / \mathrm{L}$ phenol, wherein a strain was obtained that had improved not just the tolerance to phenol but also the removal efficiency thereof. This indicates that pressure amelioration by ALE can modify multiple performance of the evolved strain simultaneously.

Through comparative transcriptomic analysis, genes that are selectively regulated under ALE conditions can be identified, and the mechanism of tolerance improvement can be inferred. A study by Zhou et al. (2017) stands as an explicit example of ALE results explicating the molecular mechanisms of adaptation: a Chlorella strain was evolved for enhanced phenol tolerance, the final biomass of the resulting strain was 1.5 times higher than that of the original strain under $500 \mathrm{mg} / \mathrm{L}$ phenol (Wang et al., 2016). The improvement of the tolerance of evolutionary strains obtained by ALE under a single stress is consistently specific to the applied stress. Using a complex mixture such as flue gas (Cheng et al., 2019), wastewater (Osundeko et al., 2014; Rezaei et al., 2019), and landfill leachate (Okurowska et al., 2021) as a complex stress condition for ALE can improve the tolerance of microalgae to different components in these mixtures simultaneously. Comparative transcriptomic analysis revealed that the genes related to superoxide dismutase (SOD), ascorbate peroxidase (APX), catalase (CAT), glutathione reductase (GR), and carotenoid biosynthesis were significantly up-regulated by $500 \mathrm{mg} / \mathrm{L}$ phenol ALE compared with the original Chlorella sp. L5 strain, indicating that ALE can allow Chlorella sp. L5 to exhibit stronger defense against antioxidants (Zhou et al., 2017).

It is worth noting that not all ALE experiments can improve the tolerance of microalgae, as has been shown in ALE of Nitzschia closterium and Chlorella sp. 12 using $\mathrm{Cu}$ (Johnson et al., 2007). This may be because the selected $\mathrm{Cu}$ concentration is too low, resulting in the low evolutionary efficiency, which is not enough to improve the tolerance to any significant extent within the duration of the experiment. Inversely, ALE with single stress can be used to enhance tolerance to multiple stress simultaneously, as proved by 
a study in which C. reinhardtii CC125 strain was evolved for enhanced tolerance to both UVR and rose Bengal (Korkaric et al., 2015).

\section{ALE APPLICATION IN REGULATING MICROALGAL METABOLIC PATHWAYS FOR PRODUCING BIOLOGICALLY ACTIVE SUBSTANCES}

Maximizing the yield of valuable target metabolites is an important issue needing to be addressed in microalgal biotechnology applications (Sun et al., 2016; Zhao et al., 2019; Deka et al., 2020). Reasonable ALE cannot only improve the tolerance of microalgae to abiotic stress, but also optimize the yield of target metabolites. ALE can increase the synthesis of high-value metabolites, including docosahexaenoic acid (DHA) (Li X. et al., 2017; Sun et al., 2018b; Diao et al., 2019; Gachelin et al., 2020; Hu et al., 2021), eicosapentaenoic acid (EPA) (Wang et al., 2019), carbohydrates (Cheng et al., 2019), carotenoid (Fu et al., 2013; Yi et al., 2015; Cheng et al., 2016; Han et al., 2019). Furthermore, the production of biofuel and high value-added by-products from ALE will improve the economic feasibility of biofuel production and microalgal biorefineries. ALE used to phenol up-regulated genes related to fatty acid and starch biosynthesis pathways, resulting in higher total carbohydrate and lipid contents than the original strain (Zhou et al., 2017). Use of glucose for ALE could increase the accumulation of DHA-rich lipids, up-regulate the hub metabolites of glycerol, glutamic acid, malonic acid, and succinic acid, and improve the negative regulation of tyrosine, fructose and lyxose (Li X. et al., 2017). By implementing ALE, Schizochytrium sp. HX-308 was evolved to increase the metabolic flux of fatty acid synthase pathway and inhibit the metabolism of polyketide synthase pathway, resulting in a 53\% higher lipid content than that when using the original strain (Sandberg et al., 2019). After ALE with 15\% $\mathrm{CO}_{2}$, the up-regulation of FBA, TPI (triosephosphate isomerase), and $P K$ (pyruvate kinase) increased the glycolysis pathways. This up-regulation aids conversion of photosynthetic carbon to pyruvate (an imperative precursor for astaxanthin and lipid synthesis) (Li K. et al., 2017).

Chemical modulators can perturb the biological system and induce cells to metabolize desired chemicals by specifically targeting enzyme proteins or as signaling molecules (Carlson and White, 2012). This strategy can be used to improve microalgal lipid production, but the direct addition of chemical modulators to the medium has obvious negative effects on biomass accumulation or lipid synthesis (Wase et al., 2017). Even so, chemical modulator-based ALE has also been profitably operated, as in a study by Diao et al. (2019) wherein the addition of acetyl-CoA carboxylase (ACCase) inhibitor could redirect carbon equivalents from starch to lipid. After sesamolbased ALE, the lipid and DHA productivities of Crypthecodinium cohnii were doubled (Diao et al., 2019). Such approaches can be used to optimize the metabolites of microalgae in various industrial applications.
Although the ability of ALE to enhance the desired microalgal metabolite production has been demonstrated, the study still indicated that there was no difference in lipid productivities between a Chlorococcum littorale strain grown after eight cycles of short-term nitrogen starvation and a control group (Cabanelas et al., 2016). The results indicated that suitable algal strains and an appropriate ALE method were also important factors to improve the metabolic yield. As the understanding of metabolic and regulatory networks continues to increase, ALE will be increasingly used as a complementary technique for optimizing high-value-added microalgae.

\section{ALE APPLICATION IN PROMOTING BIODEGRADATION OF POLLUTANTS BY MICROALGAE}

For some industrial applications such as wastewater treatment, flue gas treatment, and bio-enhanced degradation of hazardous chemicals it is necessary for microalgae to make effective use of substrates. The primary motivation of improving the substrate utilization by microalgae is usually related to the treatment of pollutants (Acuner and Dilek, 2004). The removal rate of $10 \mathrm{mg} / \mathrm{L}$ levofloxacin (LEV) by Chlorella vulgaris was increased by $67 \%$, and the half-life was shortened by 92 days, after ALE with $200 \mathrm{mg} / \mathrm{L}$ levofloxacin (Xiong et al., 2017). Phenol is the most commonly used organic contaminant for ALE of microalgae. Using 100-500 mg/L phenol for ALE can improve the removal rate of phenol and shorten its half-life in the environment when using Chlorella sp. (Wang et al., 2016) and I. galbana Parke (Li et al., 2021). ALE has been used in a trouble-shooting role in the utilization of municipal secondarytreated wastewater (Osundeko et al., 2014), sludge extracts (Wang et al., 2018), and synthetic wastewater (Rezaei et al., 2019) as a nutrient source, and can improve the removal ability of microalgae by through metabolic selection. In conclusion, ALE is suitable for use in microalgae-based wastewater treatment and cultivation without, or with reduced, dilution. Particularly, ALE successfully enhanced the removal rates of $\mathrm{SO}_{x}$, and $\mathrm{NO}_{x}$ by up-regulating genes related to extracellular sulfur transport and nitrate reductase in Chlorella sp. (Cheng et al., 2019).

\section{SUMMARY}

ALE is a promising approach to improve the phenotype of microalgae based on random mutation and natural selection. Moreover, ALE can improve the tolerance of microalgae to the selected stress conditions and the rate of substrate utilization and provide technical support for the actual production of microalgal metabolites and microalgae-based enhanced bioremediation of pollutants. Evolved strains induced by ALE have been demonstrated to activate latent metabolic pathways, enhance substrate tolerance, and improve the fitness of microalgae.

ALE also has some limitations: it can only achieve phenotype 
optimization through mutation and evolution; the evolved microalgal strains may have some side effects while improving the fitness of selection (e.g., overproduction of unnecessary metabolites, and elimination of the original fitness). Besides, the stability and reversibility of ALE resulting strains are generally not mentioned. Therefore, it is also a crucial part of the future study to verify the long-term stability of ALE resultant strains. For the design of ALE experiment, the evolution time, cycle frequency, and stress selection are the key factors for the success of ALE. In industrial /practical application, the appropriate ALE time should be selected. If ALE is too long, the modification of phenotype will gradually become insignificant, rendering the ALE uneconomic.

In conclusion, improving the efficiency of ALE is the primary problem to be solved in future research. It may be useful to solve this problem by applying multiple, simultaneous stress conditions on ALE in microalgae. As a result, ALE improves the tolerance of algal strains, optimizes the metabolites, and increases the rate of utilization of pollutants, making it a promising method for the application of microalgae biotechnology.

\section{REFERENCES}

Acuner, E., and Dilek, F. B. (2004). Treatment of tectilon yellow 2 G by Chlorella vulgaris. Process Biochem. 39, 623-631. doi: 10.1016/S0032-9592(03)00138-9

Atwood, K. C., Schneider, L. K., and Ryan, F. J. (1951). Periodic selection in Escherichia coli. Proc. Natl. Acad. Sci. U.S.A. 37, 146-155. doi: 10.1073/pnas. 37.3.146

Bautista-Chamizo, E., Borrero-Santiago, A. R., De Orte, M. R., Delvalls, Á, and Riba, I. (2018). Effects of CO2 enrichment on two microalgae species: a toxicity approach using consecutive generations. Chemosphere 213, 84-91. doi: 10.1016/ j.chemosphere.2018.09.001

Bennett, A. F., and Lenski, R. E. (1993). Evolutionary adaptation to temperature II. Thermal niches of experimental lines of Escherichia coli. Evolution 47, 1-12. doi: $10.2307 / 2410113$

Bennett, A. F., Lenski, R. E., and Mittler, J. E. (1992). Evolutionary adaptation to temperature I. Fitness responses of Escherichia coli to changes in its thermal environment. Evolution 46, 16-30. doi: 10.2307/2409801

Bittihn, P., Hasty, J., and Tsimring, L. S. (2017). Suppression of beneficial mutations in dynamic microbial populations. Phys. Rev. Lett. 118:028102. doi: 10.1103/ PhysRevLett.118.028102

Cabanelas, I. T. D., Kleinegris, D. M. M., Wijffels, R. H., and Barbosa, M. J. (2016). Repeated nitrogen starvation doesn't affect lipid productivity of Chlorococcum littorale. Bioresour. Technol. 219, 576-582. doi: 10.1016/j.biortech.2016.08.009

Carlson, S. M., and White, F. M. (2012). Expanding applications of chemical genetics in signal transduction. Cell Cycle 11, 1903-1909. doi: 10.4161/cc.19956

Cheng, D., Li, X., Yuan, Y., Yang, C., Tang, T., Zhao, Q., et al. (2019). Adaptive evolution and carbon dioxide fixation of Chlorella sp. in simulated flue gas. Sci. Total Environ. 650, 2931-2938. doi: 10.1016/j.scitotenv.2018.10.070

Cheng, J., Li, K., Yang, Z., Lu, H., Zhou, J., and Cen, K. (2016). Gradient domestication of Haematococcus pluvialis mutant with $15 \% \mathrm{CO} 2$ to promote biomass growth and astaxanthin yield. Bioresour. Technol. 216, 340-344. doi: 10.1016/j.biortech.2016.05.095

Cho, K., Lee, C. H., Ko, K., Lee, Y. J., Kim, K. N., Kim, M. K., et al. (2016). Use of phenol-induced oxidative stress acclimation to stimulate cell growth and biodiesel production by the oceanic microalga Dunaliella salina. Algal Res. 17, 61-66. doi: 10.1016/j.algal.2016.04.023

Collins, S., and Bell, G. (2004). Phenotypic consequences of 1,000 generations of selection at elevated CO2 in a green alga. Nature 431, 566-569. doi: 10.1038/ nature02945

\section{AUTHOR CONTRIBUTIONS}

BZ drafted the manuscript. All authors contributed significantly to improve the manuscript through intensive discussion and substantial additions and revisions of passages in the text. All authors contributed to the article and approved the submitted version.

\section{FUNDING}

This work was financially supported by the National Natural Science Foundation of China (No. 42077335) and the National Marine Hazard Mitigation Service, Ministry of Natural Resources of the People's Republic of China (No. 2019005AC).

\section{SUPPLEMENTARY MATERIAL}

The Supplementary Material for this article can be found online at: https://www.frontiersin.org/articles/10.3389/fmicb. 2021.737248/full\#supplementary-material

Deblois, C. P., Dufresne, K., and Juneau, P. (2013). Response to variable light intensity in photoacclimated algae and cyanobacteria exposed to atrazine. Aquat. Toxicol. 126, 77-84. doi: 10.1016/j.aquatox.2012.09.005

Deka, D., Marwein, R., Chikkaputtaiah, C., Kaki, S. S., Azmeera, T., Boruah, H. P. D., et al. (2020). Strain improvement of long-chain fatty acids producing Micractinium sp. by flow cytometry. Process Biochem. 96, 90-101. doi: 10.1016/ j.procbio.2020.06.004

Diao, J., Song, X., Cui, J., Liu, L., Shi, M., Wang, F., et al. (2019). Rewiring metabolic network by chemical modulator based laboratory evolution doubles lipid production in Crypthecodinium cohnii. Metab. Eng. 51, 88-98. doi: 10. 1016/j.ymben.2018.10.004

Fu, W., Gudmundsson, O., Feist, A. M., Herjolfsson, G., Brynjolfsson, S., and Palsson, B. $\varnothing$ (2012). Maximizing biomass productivity and cell density of Chlorella vulgaris by using light-emitting diode-based photobioreactor. J. Biotechnol. 161, 242-249. doi: 10.1016/j.jbiotec.2012.07.004

Fu, W., Guð̊mundsson, Ó, Paglia, G., Herjólfsson, G., Andrésson, ÓS., Palsson, B. $\varnothing$, et al. (2013). Enhancement of carotenoid biosynthesis in the green microalga Dunaliella salina with light-emitting diodes and adaptive laboratory evolution. Appl. Microbiol. Biotechnol. 97, 2395-2403. doi: 10.1007/s00253-012-4 502-5

Gachelin, M., Boutoute, M., Carrier, G., Talec, A., Pruvost, E., Guihéneuf, F., et al. (2020). Enhancing PUFA-rich polar lipids in Tisochrysis lutea using adaptive laboratory evolution (ALE) with oscillating thermal stress. Appl. Microbiol. Biotechnol. 105, 301-312. doi: 10.1007/s00253-020-11000-4

Gresham, D., and Dunham, M. J. (2014). The enduring utility of continuous culturing in experimental evolution. Genomics 104, 399-405. doi: 10.1016/j. ygeno.2014.09.015

Han, S. I., Kim, S., Lee, C., and Choi, Y. E. (2019). Blue-Red LED wavelength shifting strategy for enhancing beta-carotene production from halotolerant microalga, Dunaliella salina. J. Microbiol. 57, 101-106. doi: 10.1007/s12275019-8420-4

Helliwell, K. E., Collins, S., Kazamia, E., Purton, S., Wheeler, G. L., and Smith, A. G. (2015). Fundamental shift in vitamin B12 eco-physiology of a model alga demonstrated by experimental evolution. ISME J. 9, 1446-1455. doi: 10.1038/ ismej.2014.230

Hu, L., He, J., Dong, M., Tang, X., Jiang, P., Lei, A., et al. (2020). Divergent metabolic and transcriptomic responses of Synechocystis sp. PCC 6803 to salt stress after adaptive laboratory evolution. Algal Res. 47, 101856. doi: 10.1016/j. algal.2020.101856 
Hu, X., Tang, X., Bi, Z., Zhao, Q., and Ren, L. (2021). Adaptive evolution of microalgae Schizochytrium sp. under high temperature for efficient production of docosahexaeonic acid. Algal Res. 54:102212. doi: 10.1016/j.algal.2021.102212

Huertas, I. E., Rouco, M., López-Rodas, V., and Costas, E. (2011). Warming will affect phytoplankton differently: evidence through a mechanistic approach. Proc. R. Soc. B 278, 3534-3543. doi: 10.1098/rspb.2011.0160

Johnson, H. L., Stauber, J. L., Adams, M. S., and Jolley, D. F. (2007). Copper and zinc tolerance of two tropical microalgae after copper acclimation. Environ. Toxicol. 22, 234-244. doi: 10.1002/tox.20265

Kato, Y., Ho, S. H., Vavricka, C. J., Chang, J. S., Hasunuma, T., and Kondo, A. (2017). Evolutionary engineering of salt-resistant Chlamydomonas sp. strains reveals salinity stress-activated starch-to-lipid biosynthesis switching. Bioresour. Technol. 245, 1484-1490. doi: 10.1016/j.biortech.2017.06.035

Kim, Z. H., Kim, K., Park, H., Lee, C. S., Nam, S. W., Yim, K. J., et al. (2021). Enhanced fatty acid productivity by Parachlorella sp., a freshwater microalga, via adaptive laboratory evolution under salt stress. Biotechnol. Bioproc. Eng. 26, 223-231. doi: 10.1007/s12257-020-0001-1

Kleine, T., Nagele, T., Neuhaus, H. E., Schmitz-Linneweber, C., Fernie, A. R., Geigenberger, P., et al. (2021). Acclimation in plants-the green hub consortium. Plant J. 106, 23-40. doi: 10.1111/tpj.15144

Korkaric, M., Xiao, M., Behra, R., and Eggen, R. I. L. (2015). Acclimation of Chlamydomonas reinhardtii to ultraviolet radiation and its impact on chemical toxicity. Aquat. Toxicol. 167, 209-219. doi: 10.1016/j.aquatox.2015.08.008

Kuebler, J. E., Davison, I. R., and Yarish, C. (1991). Photosynthetic adaptation to temperature in the red algae Lomentaria baileyana and Lomentaria orcadensis. Eur. J. Phycol. 26, 9-19. doi: 10.1080/00071619100650021

Lachapelle, J., Bell, G., and Colegrave, N. (2015). Experimental adaptation to marine conditions by a freshwater alga. Evolution 69, 2662-2675. doi: 10.1111/ evo. 12760

LaCroix, R. A., Palsson, B. O., Feist, A. M., and Kivisaar, M. (2017). A model for designing adaptive laboratory evolution experiments. Appl. Environ. Microbiol. 83:e03115-16. doi: 10.1128/AEM.03115-16

LaPanse, A. J., Krishnan, A., and Posewitz, M. C. (2021). Adaptive laboratory evolution for algal strain improvement: methodologies and applications. Algal Res. 53:102122. doi: 10.1016/j.algal.2020.102122

Li, D., Wang, L., Zhao, Q., Wei, W., and Sun, Y. (2015). Improving high carbon dioxide tolerance and carbon dioxide fixation capability of Chlorella sp. by adaptive laboratory evolution. Bioresour. Technol. 185, 269-275. doi: 10.1016/ j.biortech.2015.03.011

Li, H., Tan, J., Sun, T., Wang, Y., and Meng, F. (2021). Acclimation of Isochrysis galbana Parke (Isochrysidaceae) for enhancing its tolerance and biodegradation to high-level phenol in seawater. Ecotoxicol. Environ. Saf. 207:111571. doi: 10.1016/j.ecoenv.2020.111571

Li, K., Cheng, J., Lu, H., Yang, W., Zhou, J., and Cen, K. (2017). Transcriptomebased analysis on carbon metabolism of Haematococcus pluvialis mutant under 15\% CO2. Bioresour. Technol. 233, 313-321. doi: 10.1016/j.biortech.2017.02. 121

Li, T., Xu, G., Rong, J., Chen, H., He, C., Giordano, M., et al. (2016). The acclimation of Chlorella to high-level nitrite for potential application in biological NOx removal from industrial flue gases. J. Plant Physiol. 195, 73-79. doi: 10.1016/j.jplph.2016.03.006

Li, X., Pei, G., Liu, L., Chen, L., and Zhang, W. (2017). Metabolomic analysis and lipid accumulation in a glucose tolerant Crypthecodinium cohnii strain obtained by adaptive laboratory evolution. Bioresour. Technol. 235, 87-95. doi: 10.1016/j.biortech.2017.03.049

Li, X., Yuan, Y., Cheng, D., Gao, J., Kong, L., Zhao, Q., et al. (2018). Exploring stress tolerance mechanism of evolved freshwater strain Chlorella sp. S30 under $30 \mathrm{~g} / \mathrm{L}$ salt. Bioresour. Technol. 250, 495-504. doi: 10.1016/j.biortech.2017.11.072

Listmann, L., Hattich, G. S. I., Matthiessen, B., and Reusch, T. B. H. (2020). Ecoevolutionary interaction in competing phytoplankton: nutrient driven genotype sorting likely explains dominance shift and species responses to CO2. Front. Mar. Sci. 7:13. doi: 10.3389/fmars.2020.00634

Lohbeck, K. T., Riebesell, U., and Reusch, T. B. H. (2012). Adaptive evolution of a key phytoplankton species to ocean acidification. Nat. Geosci. 5, 346-351. doi: 10.1038/ngeo1441

Lohbeck, K. T., Riebesell, U., and Reusch, T. B. H. (2014). Gene expression changes in the coccolithophore Emiliania huxleyi after 500 generations of selection to ocean acidification. Proc. R. Soc. B 281:20140003. doi: 10.1098/rspb.2014.0003
Marvá, F., López-Rodas, V., Rouco, M., Navarro, M., Toro, F. J., Costas, E., et al. (2010). Adaptation of green microalgae to the herbicides simazine and diquat as result of pre-selective mutations. Aquat. Toxicol. 96, 130-134. doi: 10.1016/j. aquatox.2009.10.009

Meijer, H. J. G., Himbergen, J. A. J. V., Musgrave, A., and Munnik, T. (2017). Acclimation to salt modifies the activation of several osmotic stress-activated lipid signalling pathways in Chlamydomonas. Phytochemistry 135, 64-72. doi: 10.1016/j.phytochem.2016.12.014

Mikulic, P., and Beardall, J. (2014). Contrasting ecotoxicity effects of zinc on growth and photosynthesis in a neutrophilic alga (Chlamydomonas reinhardtii) and an extremophilic alga (Cyanidium caldarium). Chemosphere 112, 402-411. doi: 10.1016/j.chemosphere.2014.04.049

Mohsenpour, S. F., Hennige, S., Willoughby, N., Adeloye, A., and Gutierrez, T. (2021). Integrating micro-algae into wastewater treatment: a review. Sci. Total Environ. 752:142168. doi: 10.1016/j.scitotenv.2020.142168

Muyssen, B. T. A., and Janssen, C. R. (2001). Zinc acclimation and its effect on the zinc tolerance of Raphidocelis subcapitata and Chlorella vulgaris in laboratory experiments. Chemosphere 45, 507-514. doi: 10.1016/S0045-6535(01)00047-9

Novick, A., and Szilard, L. (1950). Experiments with the chemostat on spontaneous mutations of bacteria. Proc. Natl. Acad. Sci. U.S.A. 36, 708-719. doi: 10.1073/ pnas.36.12.708

Okurowska, K., Karunakaran, E., Al-Farttoosy, A., Couto, N., and Pandhal, J. (2021). Adapting the algal microbiome for growth on domestic landfill leachate. Bioresour. Technol. 319:124246. doi: 10.1016/j.biortech.2020.124246

Osundeko, O., Dean, A. P., Davies, H., and Pittman, J. K. (2014). Acclimation of microalgae to wastewater environments involves increased oxidative stress tolerance activity. Plant Cell Physiol. 55, 1848-1857. doi: 10.1093/pcp/pcu113

Ownby, D. R., Newman, M. C., Mulvey, M., Vogelbein, W. K., Unger, M. A., and Arzayus, L. F. (2002). Fish (Fundulus heteroclitus) populations with different exposure histories differ in tolerance of creosote-contaminated sediments. Environ. Toxicol. Chem. 21, 1897-1902. doi: 10.1002/etc.5620210917

Perrineau, M. M., Gross, J., Zelzion, E., Price, D. C., Levitan, O., Boyd, J., et al. (2014a). Using natural selection to explore the adaptive potential of Chlamydomonas reinhardtii. PLoS One 9:e92533. doi: 10.1371/journal.pone. 0092533

Perrineau, M. M., Zelzion, E., Gross, J., Price, D. C., Boyd, J., and Bhattacharya, D. (2014b). Evolution of salt tolerance in a laboratory reared population of Chlamydomonas reinhardtii. Environ. Microbiol. 16, 1755-1766. doi: 10.1111/ 1462-2920.12372

Qi, F., Zhang, M., Chen, Y., Jiang, X., Lin, J., Cao, X., et al. (2017). A lignocellulosic hydrolysate-tolerant Aurantiochytrium sp. mutant strain for docosahexaenoic acid production. Bioresour. Technol. 227, 221-226. doi: 10.1016/j.biortech.2016. 12.011

Rezaei, R., Akbulut, A., and Sanin, S. L. (2019). Effect of algae acclimation to the wastewater medium on the growth kinetics and nutrient removal capacity. Environ. Monit. Assess. 191:679. doi: 10.1007/s10661-019-7856-7

Rossoni, A. W., and Weber, A. P. M. (2019). Systems biology of cold adaptation in the polyextremophilic red alga Galdieria sulphuraria. Front. Microbiol. 10:14. doi: $10.3389 /$ fmicb. 2019.00927

Sandberg, T. E., Salazar, M. J., Weng, L. L., Palsson, B. O., and Feist, A. M. (2019). The emergence of adaptive laboratory evolution as an efficient tool for biological discovery and industrial biotechnology. Metab. Eng. 56, 1-16. doi: 10.1016/j.ymben.2019.08.004

Schaum, C. E., Barton, S., Bestion, E., Buckling, A., Garcia-Carreras, B., Lopez, P., et al. (2017). Adaptation of phytoplankton to a decade of experimental warming linked to increased photosynthesis. Nat. Ecol. Evol. 1:0094. doi: 10.1038/s41559017-0094

Schlüter, L., Lohbeck, K. T., Gutowska, M. A., Gröger, J. P., Riebesell, U., and Reusch, T. B. H. (2014). Adaptation of a globally important coccolithophore to ocean warming and acidification. Nat. Clim. Change 4, 1024-1030. doi: $10.1038 /$ nclimate2379

Shin, S. E., Koh, H. G., Kang, N. K., Suh, W. I., Jeong, B. R., Lee, B., et al. (2017). Isolation, phenotypic characterization and genome wide analysis of a Chlamydomonas reinhardtii strain naturally modified under laboratory conditions: towards enhanced microalgal biomass and lipid production for biofuels. Biotechnol. Biofuels 10:308. doi: 10.1186/s13068-017-1000-0

Sun, X. M., Ren, L. J., Bi, Z. Q., Ji, X. J., Zhao, Q. Y., and Huang, H. (2018a). Adaptive evolution of microalgae Schizochytrium sp. under high salinity 
stress to alleviate oxidative damage and improve lipid biosynthesis. Bioresour. Technol. 267, 438-444. doi: 10.1016/j.biortech.2018.07.079

Sun, X. M., Ren, L. J., Bi, Z. Q., Ji, X. J., Zhao, Q. Y., Jiang, L., et al. (2018b). Development of a cooperative two-factor adaptive-evolution method to enhance lipid production and prevent lipid peroxidation in Schizochytrium sp. Biotechnol. Biofuels. 11:65. doi: 10.1186/s13068-018-1065-4

Sun, X. M., Ren, L. J., Ji, X. J., Chen, S. L., Guo, D. S., and Huang, H. (2016). Adaptive evolution of Schizochytrium sp. by continuous high oxygen stimulations to enhance docosahexaenoic acid synthesis. Bioresour. Technol. 211, 374-381. doi: 10.1016/j.biortech.2016.03.093

Wang, L., Wang, H., Chen, X., Zhuang, Y., Yu, Z., and Zhou, T. (2018). Acclimation process of cultivating Chlorella vulgaris in toxic excess sludge extract and its response mechanism. Sci. Total Environ. 628-629, 858-869. doi: 10.1016/j. scitotenv.2018.02.020

Wang, L., Xue, C., Wang, L., Zhao, Q., Wei, W., and Sun, Y. (2016). Strain improvement of Chlorella sp. for phenol biodegradation by adaptive laboratory evolution. Bioresour. Technol. 205, 264-268. doi: 10.1016/j.biortech.2016.01. 022

Wang, X., Luo, S. W., Luo, W., Yang, W. D., Liu, J. S., and Li, H. Y. (2019). Adaptive evolution of microalgal strains empowered by fulvic acid for enhanced polyunsaturated fatty acid production. Bioresour. Technol. 277, 204-210. doi: 10.1016/j.biortech.2018.12.116

Wase, N., Tu, B., Allen, J. W., Black, P. N., and Dirusso, C. C. (2017). Identification and metabolite profiling of chemical activators of lipid accumulation in green algae. Plant Physiol. 174, 2146-2165. doi: 10.1104/pp.17.00433

Xiong, J. Q., Kurade, M. B., and Jeon, B. H. (2017). Biodegradation of levofloxacin by an acclimated freshwater microalga, Chlorella vulgaris. Chem. Eng. J. 313, 1251-1257. doi: 10.1016/j.cej.2016.11.017

Yi, Z., Xu, M., Magnusdottir, M., Zhang, Y., Brynjolfsson, S., and Fu, W. (2015). Photo-oxidative stress-driven mutagenesis and adaptive evolution on the marine diatom Phaeodactylum tricornutum for enhanced carotenoid accumulation. Mar. Drugs 13, 6138-6151. doi: 10.3390/md13106138

Yu, S., Zhao, Q., Miao, X., and Shi, J. (2013). Enhancement of lipid production in low-starch mutants Chlamydomonas reinhardtii by adaptive laboratory evolution. Bioresour. Technol. 147, 499-507. doi: 10.1016/j.biortech.2013.08. 069

Yu, Z., Zhang, T., and Zhu, Y. (2020). Whole-genome re-sequencing and transcriptome reveal cadmium tolerance related genes and pathways in Chlamydomonas reinhardtii. Ecotoxicol. Environ. Saf. 191:110231. doi: 10.1016/ j.ecoenv.2020.110231

Zchut, S., Keren, N. I. R., Ohad, I., and Pick, U. R. I. (2003). Cold-acclimation protects photosystem II against freezing damage in the halotolerant alga
Dunaliella salina. J. Plant Physiol. 160, 185-192. doi: 10.1078/0176-1617-0 0723

Zhang, Y., Gu, Z., Ren, Y., Wang, L., Zhang, J., Liang, C., et al. (2021). Integrating transcriptomics and metabolomics to characterize metabolic regulation to elevated CO2 in Chlamydomonas Reinhardtii. Mar. Biotechnol. 23, 255-275. doi: 10.1007/s10126-021-10021-y

Zhao, Q., and Huang, H. (2020). Adaptive evolution improves algal strains for environmental remediation. Trends Biotechnol. 39, 112-115. doi: 10.1016/j.tibtech.2020.0 8.009

Zhao, Y., Wang, H. P., Han, B., and Yu, X. (2019). Coupling of abiotic stresses and phytohormones for the production of lipids and high-value by-products by microalgae: a review. Bioresour. Technol. 274, 549-556. doi: 10.1016/j.biortech. 2018.12.030

Zhong, J., Guo, Y., Liang, Z., Huang, Q., Lu, H., Pan, J., et al. (2021). Adaptation of a marine diatom to ocean acidification and warming reveals constraints and trade-offs. Sci. Total Environ. 771:145167. doi: 10.1016/j.scitotenv.2021.145167

Zhou, L., Cheng, D., Wang, L., Gao, J., Zhao, Q., Wei, W., et al. (2017). Comparative transcriptomic analysis reveals phenol tolerance mechanism of evolved Chlorella strain. Bioresour. Technol. 227, 266-272. doi: 10.1016/j. biortech.2016.12.059

Zhu, Q. L., Bao, J., Liu, J., and Zheng, J. L. (2020). High salinity acclimatization alleviated cadmium toxicity in Dunaliella salina: transcriptomic and physiological evidence. Aquat. Toxicol. 223:105492. doi: 10.1016/j.aquatox.2020.105492

Conflict of Interest: The authors declare that the research was conducted in the absence of any commercial or financial relationships that could be construed as a potential conflict of interest.

Publisher's Note: All claims expressed in this article are solely those of the authors and do not necessarily represent those of their affiliated organizations, or those of the publisher, the editors and the reviewers. Any product that may be evaluated in this article, or claim that may be made by its manufacturer, is not guaranteed or endorsed by the publisher.

Copyright (c) 2021 Zhang, Wu and Meng. This is an open-access article distributed under the terms of the Creative Commons Attribution License (CC BY). The use, distribution or reproduction in other forums is permitted, provided the original author(s) and the copyright owner(s) are credited and that the original publication in this journal is cited, in accordance with accepted academic practice. No use, distribution or reproduction is permitted which does not comply with these terms. 